\title{
ON CONGRUENCES WITH THE TERMS OF SECOND ORDER SEQUENCE $\left\{\boldsymbol{Q}_{n}\right\}$
}

\section{NEŞE ÖMÜR and SIBEL KOPARAL}

Department of Mathematics

Kocaeli University

41380 Izmit Kocaeli

Turkey

e-mail: neseomur@kocaeli.edu.tr

sibel.koparal@kocaeli.edu.tr

$$
\begin{aligned}
& \text { Abstract } \\
& \text { In this paper, we obtain various basic congruences involving harmonic numbers } \\
& \text { and the terms of the second order sequence }\left\{Q_{n}\right\} \text {. For example, for even } t \text { and } \\
& \left(\frac{t}{3}\right) \neq 0 \\
& -3 \sum_{k=1}^{p-1} H_{k} Q_{t k+d} \\
& \equiv \frac{p B_{p-2}\left(\frac{1}{3}\right)}{3(p-2)} Q_{d+(2-2 \delta) t}+\frac{3}{2}\left(\frac{p}{3}\right) Q_{d+2 \delta t}\left(q_{p}(3)-\frac{1}{2} p q_{p}^{2}(3)\right)\left(\bmod p^{2}\right) \\
& \text { and for }\left(\frac{t}{3}\right)=0 \\
& \frac{1}{Q_{d}} \sum_{k=1}^{p-1} k H_{k} Q_{t k+d}
\end{aligned}
$$

2010 Mathematics Subject Classification: 11B50, 11A07, $11 \mathrm{~B} 68$.

Keywords and phrases: congruences, second order sequences, harmonic numbers.

Received January 28, 2016

(C) 2016 Scientific Advances Publishers 


$$
\begin{aligned}
\equiv \frac{p}{4}(3-\Delta(t)(3 & \left.\left.+q_{p}^{2}(2)\right)\right) \\
& -\frac{1}{2}\left(1-\Delta(t)\left(1+q_{p}(2)\right)\right)\left(\bmod p^{2}\right),
\end{aligned}
$$

where any prime $p>3, \Delta(t)=\frac{1-(-1)^{t}}{2}$, Fermat quotient $q_{p}(2)=\frac{2^{p-1}-1}{p}$,

$\delta=\frac{1-\left(\frac{p}{3}\right)}{2}$ and $(-)$ is the Jacobi symbol.

\section{Introduction}

The second order sequence $\left\{Q_{n}\right\}$ is defined by for $a, b \in \mathbb{Z}$, and $n>0$

$$
Q_{n+1}=Q_{n}-Q_{n-1},
$$

in which $Q_{0}=a, Q_{1}=b$. The characteristic equation $x^{2}-x+1=0$ of the sequence $\left\{Q_{n}\right\}$ has two roots

$$
\alpha=\frac{1+\sqrt{-3}}{2} \text { and } \beta=\frac{1-\sqrt{-3}}{2} \text {. }
$$

The Binet formula of the sequence $\left\{Q_{n}\right\}$ is

$$
Q_{n}=\frac{(b-a \beta) \alpha^{n}-(b-a \alpha) \beta^{n}}{\alpha-\beta} .
$$

The sequence $\left\{Q_{n}\right\}$ is cyclic of order 6 (with terms $a, b,-a+b,-a,-b$, $a-b)$ since $\alpha^{3 n}=\beta^{3 n}=-1$. Thus $\alpha^{6 n}=\beta^{6 n}=1$.

Harmonic numbers are those rational numbers given by

$$
H_{0}=0, \quad H_{n}=\sum_{k=1}^{n} \frac{1}{k}, \quad n \in \mathbb{N}=\{1,2, \ldots\} .
$$

Wolstenholme [2] proved that if $p>3$ is a prime, then

$$
H_{p-1} \equiv 0\left(\bmod p^{2}\right)
$$


Bernoulli numbers $\left\{B_{n}\right\}$ and Bernoulli polynomials $\left\{B_{n}(x)\right\}$ are defined by

$$
\begin{gathered}
B_{0}=1 \text { and } \sum_{k=0}^{n-1}\left(\begin{array}{l}
n \\
k
\end{array}\right) B_{k}=0(n=2,3,4, \ldots), \\
B_{n}(x)=\sum_{k=0}^{n}\left(\begin{array}{l}
n \\
k
\end{array}\right) B_{k} x^{n-k}(n=0,1,2, \ldots),
\end{gathered}
$$

respectively. The first few Bernoulli numbers are $1,-\frac{1}{2}, \frac{1}{6}, 0,-\frac{1}{30}, \ldots$ In [3], for $x \in \mathbb{R}$ and $n \in \mathbb{N}$,

$$
B_{n}(1-x)=(-1)^{n} B_{n}(x)
$$

It is known that

$$
\begin{aligned}
& B_{2 n}\left(\frac{1}{3}\right)=B_{2 n}\left(\frac{2}{3}\right)=\frac{3-3^{2 n}}{2.3^{2 n}} B_{2 n} \\
& B_{2 n}\left(\frac{1}{6}\right)=B_{2 n}\left(\frac{5}{6}\right)=\frac{\left(2-2^{2 n}\right)\left(3-3^{2 n}\right)}{2.6^{2 n}} B_{2 n}
\end{aligned}
$$

Sun [5] proved that for $b \geq 2$,

$$
\frac{B_{k(p-1)+b}(x)}{k(p-1)+b} \equiv \frac{B_{b}(x)}{b}(\bmod p)
$$

where an odd prime $p, x \in \mathbb{Z}_{p}$ and $k, b \in \mathbb{N}$ with $p-1 \nmid b$.

For a prime $p>3$ and $r \in \mathbb{Z}, k, m \in \mathbb{N}$ with $k<p-3$ and $p \nmid m$. Sun [6] gave

$$
\begin{aligned}
\sum_{\substack{x=1 \\
x \equiv r(\bmod m)}}^{p-1} \frac{1}{x^{k} \equiv} & \frac{B_{\varphi\left(p^{3}\right)-k+1}\left(\left\{\frac{r-p}{m}\right\}\right)-B_{\varphi\left(p^{3}\right)-k+1}\left(\left\{\frac{r}{m}\right\}\right)}{m^{k}\left(\varphi\left(p^{3}\right)-k+1\right)} \\
& +\frac{k p}{m^{k+1}}\left(\frac{B_{2 p-2-k}\left(\left\{\frac{r-p}{m}\right\}\right)}{2 p-2-k}-2 \frac{B_{p-1-k}\left(\left\{\frac{r-p}{m}\right\}\right)}{p-1-k}\right)
\end{aligned}
$$




$$
-\frac{k(k+1) p^{2}}{2(k+2) m^{k+2}} B_{p-2-k}\left(\left\{\frac{r-p}{m}\right\}\right)\left(\bmod p^{3}\right),
$$

where $\{x\}$ is the fractional part of $x$ and $\varphi(n)$ is Euler's totient function.

Lehmer [1] showed that for $p>3$,

$$
\sum_{k=1}^{\left\lfloor\frac{p}{3}\right\rfloor} \frac{1}{p-3 k} \equiv \frac{1}{2}\left(q_{p}(3)-\frac{1}{2} p q_{p}^{2}(3)\right)\left(\bmod p^{2}\right)
$$

and for $p>5$,

$$
\sum_{k=1}^{\left\lfloor\frac{p}{6}\right\rfloor} \frac{1}{p-6 k} \equiv \frac{1}{3}\left(q_{p}(2)-\frac{1}{2} p q_{p}^{2}(2)\right)+\frac{1}{4}\left(q_{p}(3)-\frac{1}{2} p q_{p}^{2}(3)\right)\left(\bmod p^{2}\right)
$$

Sun [8] clearly gave that for any odd prime $p$ and $k \in\{0,1, \ldots, p-1\}$,

$$
(-1)^{k}\left(\begin{array}{c}
p-1 \\
k
\end{array}\right)=\prod_{j=1}^{k}\left(1-\frac{p}{j}\right) \equiv 1-p H_{k}\left(\bmod p^{2}\right)
$$

In this paper, we obtain various basic congruences involving harmonic numbers and the terms of the second order sequence $\left\{Q_{n}\right\}$. For example, for even $t$ and $\left(\frac{t}{3}\right) \neq 0$,

$$
\begin{aligned}
& -3 \sum_{k=1}^{p-1} H_{k} Q_{t k+d} \\
& \quad \equiv \frac{p B_{p-2}\left(\frac{1}{3}\right)}{3(p-2)} Q_{d+(2-2 \delta) t}+\frac{3}{2}\left(\frac{p}{3}\right) Q_{d+2 \delta t}\left(q_{p}(3)-\frac{1}{2} p q_{p}^{2}(3)\right)\left(\bmod p^{2}\right),
\end{aligned}
$$

and for $\left(\frac{t}{3}\right)=0$, 
$\frac{1}{Q_{d}} \sum_{k=1}^{p-1} k H_{k} Q_{t k+d}$

$$
\equiv \frac{p}{4}\left(3-\Delta(t)\left(3+q_{p}^{2}(2)\right)\right)-\frac{1}{2}\left(1-\Delta(t)\left(1+q_{p}(2)\right)\right)\left(\bmod p^{2}\right),
$$

where any prime $\quad p>3, \Delta(t)=\frac{1-(-1)^{t}}{2}, \quad$ Fermat quotient $q_{p}(2)=\frac{2^{p-1}-1}{p}, \delta=\frac{1-\left(\frac{p}{3}\right)}{2}$ and $(-)$ is the Jacobi symbol.

\section{On Congruences with Second Order Sequence $\left\{Q_{n}\right\}$}

In this section, we will investigate certain congruences consisting of harmonic numbers and the terms of the second order sequence $\left\{Q_{n}\right\}$.

Lemma 1. For $n, t, d \in \mathbb{Z}$, then

$$
Q_{n t+d}=\left\{\begin{array}{cc}
(-1)^{t+1} Q_{(n-1) t+d}-Q_{(n-2) t+d}, & \text { if }\left(\frac{t}{3}\right) \neq 0, \\
(-1)^{n t} Q_{d}, & \text { if }\left(\frac{t}{3}\right)=0 .
\end{array}\right.
$$

Proof. The proof is clearly obtained from the Binet formula of the sequence $\left\{Q_{n}\right\}$.

Lemma 2 ([6]). Let $p$ be an odd prime. For $n \in \mathbb{N}$ with $p>n+1$. Then

$$
\frac{a^{\varphi\left(p^{n}\right)}-1}{p^{n}} \equiv \sum_{s=1}^{n} \frac{(-1)^{s-1}}{s} p^{s-1}\left(q_{p}(a)\right)^{s}\left(\bmod p^{n}\right),
$$

where $a \in \mathbb{Z}_{p}$ with $p \nmid a$. 
Lemma 3. Let $p>3$ be an odd prime. Then

$$
\begin{aligned}
\sum_{k=1}^{\left\lfloor\frac{p}{3}\right\rfloor} \frac{1}{p-3 k+1}+\frac{p}{9} \frac{B_{p-2}\left(\frac{1}{3}\right)}{p-2} & \text { if }\left(\frac{p}{3}\right)=1 \\
& \equiv\left\{\begin{array}{cc}
0, & \text { if }\left(\frac{p}{3}\right)=-1 \\
-\frac{1}{2}\left(q_{p}(3)-\frac{1}{2} p q_{p}^{2}(3)\right), & \left.p^{2}\right),
\end{array}\right.
\end{aligned}
$$

$$
\begin{aligned}
& \sum_{k=1}^{\left\lfloor\frac{p}{3}\right\rfloor} \frac{1}{p-3 k+2}-\frac{p}{9} \frac{B_{p-2}\left(\frac{1}{3}\right)}{p-2} \\
& \equiv\left\{\begin{array}{cl}
-\frac{1}{2}\left(q_{p}(3)-\frac{1}{2} p q_{p}^{2}(3)\right), & \text { if }\left(\frac{p}{3}\right)=1 \\
-1, & \text { if }\left(\frac{p}{3}\right)=-1
\end{array}\left(\bmod p^{2}\right),\right.
\end{aligned}
$$

$$
\begin{aligned}
& -12 \sum_{k=1}^{\left\lfloor\frac{p}{6}\right\rfloor} \frac{1}{p-6 k+1}-\frac{5 p}{3} \frac{B_{p-2}\left(\frac{1}{3}\right)}{p-2} \\
& \equiv\left\{\begin{array}{cl}
6\left(q_{p}(2)-\frac{1}{2} p q_{p}^{2}(2)\right) & \\
+2\left(q_{p}(3)-\frac{1}{2} p q_{p}^{2}(3)-q_{p}(6)+\frac{1}{2} p q_{p}^{2}(6)\right), & \text { if }\left(\frac{p}{3}\right)=1 \\
3\left(q_{p}(2)-\frac{1}{2} p q_{p}^{2}(2)\right) & \left(\bmod p^{2}\right), \\
+2\left(q_{p}(3)-\frac{1}{2} p q_{p}^{2}(3)\right)+q_{p}(6)-\frac{1}{2} p q_{p}^{2}(6), & \text { if }\left(\frac{p}{3}\right)=-1
\end{array}\right.
\end{aligned}
$$




$$
\begin{aligned}
& 12 \sum_{k=1}^{\left\lfloor\frac{p}{6}\right\rfloor} \frac{1}{p-6 k+2}+\frac{p}{3} \frac{B_{p-2}\left(\frac{1}{3}\right)}{p-2} \\
& \equiv\left\{\begin{array}{cl}
3\left(q_{p}(2)-\frac{1}{2} p q_{p}^{2}(2)\right) & \\
-4\left(q_{p}(3)-\frac{1}{2} p q_{p}^{2}(3)\right)+\left(q_{p}(6)-\frac{1}{2} p q_{p}^{2}(6)\right), & \text { if }\left(\frac{p}{3}\right)=1 \\
-12+6\left(q_{p}(2)-\frac{1}{2} p q_{p}^{2}(2)\right) & \left(\bmod p^{2}\right), \\
+2\left(q_{p}(3)-\frac{1}{2} p q_{p}^{2}(3)-q_{p}(6)+\frac{1}{2} p q_{p}^{2}(6)\right), & \text { if }\left(\frac{p}{3}\right)=-1
\end{array}\right.
\end{aligned}
$$

Proof. For $\left(\frac{p}{3}\right)=1$, by congruence in (1.5), we write

$$
\begin{aligned}
\sum_{k=1}^{\left\lfloor\frac{p}{6}\right\rfloor} \frac{1}{p-6 k+1}= & \sum_{\substack{k=1 \\
k \equiv 2(\bmod 6)}}^{p-1} \frac{1}{k} \\
\equiv & \frac{B_{p^{2}(p-1)}\left(\left\{\frac{2-p}{6}\right\}\right)-B_{p^{2}(p-1)}\left(\frac{1}{3}\right)}{6 p^{2}(p-1)} \\
& +\frac{p}{36}\left(\frac{B_{2 p-3}\left(\left\{\frac{2-p}{6}\right\}\right)}{2 p-3}-2 \frac{\left.B_{p-2}\left(\left\{\frac{2-p}{6}\right\}\right)\right)}{p-2}\right) \\
= & \frac{B_{p^{2}(p-1)}\left(\frac{1}{6}\right)-B p^{2}(p-1)\left(\frac{1}{3}\right)}{6 p^{2}(p-1)} \\
& +\frac{p}{36}\left(\frac{B_{2 p-3}\left(\frac{1}{6}\right)}{2 p-3}-2 \frac{B_{p-2}\left(\frac{1}{6}\right)}{p-2}\right)\left(\bmod p^{2}\right) .
\end{aligned}
$$

Since $p B_{p^{2}(p-1)} \equiv p-1\left(\bmod p^{3}\right)$ in [4], using (1.3), (1.4), (2.2) and Euler's theorem, we have 


$$
\begin{aligned}
& \sum_{k=1}^{\left\lfloor\frac{p}{6}\right\rfloor} \frac{1}{p-6 k+1} \\
\equiv & \left(\frac{\left(2-2^{p^{2}(p-1)}\right)\left(3-3^{p^{2}(p-1)}\right)}{2.6^{p^{2}(p-1)}}-\frac{3-3^{p^{2}(p-1)}}{2.3^{p^{2}(p-1)}}\right) \frac{B_{p^{2}(p-1)}}{6 p^{2}(p-1)}-\frac{p}{36} \frac{B_{p-2}\left(\frac{1}{6}\right)}{p-2} \\
= & \frac{1}{6^{p^{2}(p-1)}}\left(3 \frac{1-2^{p^{2}(p-1)}}{p^{3}}+\frac{1-3^{p^{2}(p-1)}}{p^{3}}+\frac{\left.6^{p^{2}(p-1)}-1\right)}{p^{3}}\right) \\
& \times \frac{p B}{6(p-1)}-\frac{p}{36} \frac{B_{p-2}\left(\frac{1}{6}\right)}{p-2} \\
\equiv & -\frac{p}{36} \frac{B_{p-2}\left(\frac{1}{6}\right)}{p-2}-\frac{1}{2}\left(q_{p}(2)-\frac{1}{2} p q_{p}^{2}(2)\right) \\
& -\frac{1}{6}\left(q_{p}(3)-\frac{1}{2} p q_{p}^{2}(3)-q_{p}(6)+\frac{1}{2} p q_{p}^{2}(6)\right)\left(\bmod p^{2}\right) .
\end{aligned}
$$

Similarly for $\left(\frac{p}{3}\right)=-1$, using the congruence in (1.5) and $p B_{p^{2}(p-1)} \equiv$ $p-1\left(\bmod p^{3}\right)$ in [4], $B_{n}(0)=B_{n}$ for $n \in \mathbb{N}, \quad(1.3), \quad(1.4), \quad(2.2)$ and Euler's theorem, we get

$$
\begin{aligned}
\sum_{k=1}^{\left\lfloor\frac{p}{6}\right\rfloor} \frac{1}{p-6 k+1} \equiv & -\frac{p}{36} \frac{B_{p-2}\left(\frac{1}{6}\right)}{p-2}-\frac{1}{4}\left(q_{p}(2)-\frac{1}{2} p q_{p}^{2}(2)\right) \\
& -\frac{1}{6}\left(q_{p}(3)-\frac{1}{2} p q_{p}^{2}(3)\right)-\frac{1}{12}\left(q_{p}(6)+\frac{1}{2} p q_{p}^{2}(6)\right)\left(\bmod p^{2}\right)
\end{aligned}
$$

Using the congruence $p B_{p-2}\left(\frac{1}{6}\right) \equiv 5 p B_{p-2}\left(\frac{1}{3}\right)\left(\bmod p^{2}\right)$ in [6], from (2.7) and (2.8), the proof is completed. 
The proof of the others are obtained similar to the proof of (2.5).

Lemma 4. Let $p>3$ be an odd prime. Then

$$
\begin{aligned}
& -\frac{3}{2} \sum_{\substack{k=1 \\
3 \mid k-p}}^{p-1} \frac{(-1)^{k}}{k} \equiv q_{p}(2)-\frac{1}{2} p q_{p}^{2}(2)\left(\bmod p^{2}\right), \\
& 3 \sum_{\substack{k=1 \\
3 \mid k-p+1}}^{p-1} \frac{(-1)^{k}}{k}-\frac{p}{2} \frac{B_{p-2}\left(\frac{1}{3}\right)}{p-2} \\
& \equiv\left\{\begin{array}{c}
-\frac{3}{2}\left(q_{p}(2)-\frac{1}{2} p q_{p}^{2}(2)\right) \\
+\frac{1}{2}\left(q_{p}(3)-\frac{1}{2} p q_{p}^{2}(3)-q_{p}(6)+\frac{1}{2} p q_{p}^{2}(6)\right), \quad \text { if }\left(\frac{p}{3}\right)=1 \\
3-3\left(q_{p}(2)-\frac{1}{2} p q_{p}^{2}(2)\right) \\
-q_{p}(3)+\frac{1}{2} p q_{p}^{2}(3)+q_{p}(6)-\frac{1}{2} p q_{p}^{2}(6), \quad \text { if }\left(\frac{p}{3}\right)=-1
\end{array}\right. \\
& 3 \sum_{\substack{k=1 \\
3 \mid k-p+2}}^{p-1} \frac{(-1)^{k}}{k}+\frac{p}{2} \frac{B_{p-2}\left(\frac{1}{3}\right)}{p-2} \\
& \equiv\left\{\begin{array}{c}
-3\left(q_{p}(2)-\frac{1}{2} p q_{p}^{2}(2)\right) \\
-q_{p}(3)+\frac{1}{2} p q_{p}^{2}(3)+q_{p}(6)-\frac{1}{2} p q_{p}^{2}(6), \quad \text { if }\left(\frac{p}{3}\right)=1 \\
\quad-\frac{3}{2}\left(q_{p}(2)-\frac{1}{2} p q_{p}^{2}(2)\right) \\
+\frac{1}{2}\left(q_{p}(3)-\frac{1}{2} p q_{p}^{2}(3)-q_{p}(6)+\frac{1}{2} p q_{p}^{2}(6)\right), \quad \text { if }\left(\frac{p}{3}\right)=-1
\end{array}\right.
\end{aligned}
$$


Proof. We can give proof of (2.10).

By [7], it is known that

$$
3 \sum_{\substack{k=1 \\ 3 \mid k-p}}^{p-1} \frac{(-1)^{k}}{k} \equiv \sum_{k=1}^{p-1} \frac{(-1)^{k}}{k}-\sum_{k=1}^{\frac{p-1}{2}} \frac{1}{k}+3 \sum_{k=1}^{\left\lfloor\frac{p}{3}\right\rfloor} \frac{1}{p-3 k}-6 \sum_{k=1}^{\left\lfloor\frac{p}{6}\right\rfloor} \frac{1}{p-6 k}\left(\bmod p^{2}\right) .
$$

We can also easily show

$$
\begin{aligned}
3 \sum_{\substack{k=1 \\
3 \mid k-p+1}}^{p-1} \frac{(-1)^{k}}{k} \equiv \sum_{k=1}^{p-1} \frac{(-1)^{k}}{k}-\sum_{k=1}^{\frac{p-1}{2}} \frac{1}{k}+3 \sum_{k=1}^{\left\lfloor\frac{p}{3}\right\rfloor} \frac{1}{p-3 k+2} \\
\\
-6 \sum_{k=1}^{\left\lfloor\frac{p}{6}\right\rfloor} \frac{1}{p-6 k+2}\left(\bmod p^{2}\right),
\end{aligned}
$$

and

$$
\begin{aligned}
3 \sum_{\substack{k=1 \\
3 \mid k-p+2}}^{p-1} \frac{(-1)^{k+1}}{k} \equiv & \sum_{k=1}^{p-1} \frac{(-1)^{k}}{k}-\sum_{k=1}^{\frac{p-1}{2}} \frac{1}{k}+3 \sum_{k=1}^{\left\lfloor\frac{p}{3}\right\rfloor} \frac{1}{p-3 k+1} \\
& -6 \sum_{k=1}^{\left\lfloor\frac{p}{6}\right\rfloor} \frac{1}{p-6 k+1}\left(\bmod p^{2}\right) .
\end{aligned}
$$

For $\left(\frac{p}{3}\right)=1$, by the congruences $\sum_{j=1}^{(p-1) / 2} \frac{1}{j} \equiv-2\left(q_{p}(2)-\frac{1}{2} p q_{p}^{2}(2)\right)\left(\bmod p^{2}\right)$ in [1] and $\sum_{j=1}^{p-1} \frac{(-1)^{j}}{j} \equiv-2\left(q_{p}(2)-\frac{1}{2} p q_{p}^{2}(2)\right)\left(\bmod p^{2}\right)$ in [6]; using (2.4) and (2.6), we write 


$$
\begin{aligned}
3 \sum_{\substack{k=1 \\
3 \mid k-p+1}}^{p-1} \frac{(-1)^{k}}{k} & \\
\equiv & \frac{p}{3} \frac{B_{p-2}\left(\frac{1}{3}\right)}{p-2}-\frac{3}{2}\left(q_{p}(3)-\frac{1}{2} p q_{p}^{2}(3)\right)+\frac{p}{6} \frac{B_{p-2}\left(\frac{1}{3}\right)}{p-2} \\
& -\frac{3}{2}\left(q_{p}(2)-\frac{1}{2} p q_{p}^{2}(2)\right)+2\left(q_{p}(3)-\frac{1}{2} p q_{p}^{2}(3)\right) \\
& -\frac{1}{2}\left(q_{p}(6)-\frac{1}{2} p q_{p}^{2}(6)\right) \\
= & \frac{p}{2} \frac{B_{p-2}\left(\frac{1}{3}\right)}{p-2}-\frac{3}{2}\left(q_{p}(2)-\frac{1}{2} p q_{p}^{2}(2)\right) \\
& +\frac{1}{2}\left(q_{p}(3)-\frac{1}{2} p q_{p}^{2}(3)\right)-\frac{1}{2}\left(q_{p}(6)-\frac{1}{2} p q_{p}^{2}(6)\right)\left(\bmod p^{2}\right) .
\end{aligned}
$$

The proof for $\left(\frac{p}{3}\right)=-1$ is similar to the proof for $\left(\frac{p}{3}\right)=1$. Thus

$$
\begin{aligned}
3 \sum_{\substack{k=1 \\
3 \mid k-p+1}}^{p-1} \frac{(-1)^{k}}{k} \equiv & \frac{p}{2} \frac{B_{p-2}\left(\frac{1}{3}\right)}{p-2}+3-3\left(q_{p}(2)-\frac{1}{2} p q_{p}^{2}(2)\right) \\
& -q_{p}(3)+\frac{1}{2} p q_{p}^{2}(3)+q_{p}(6)-\frac{1}{2} p q_{p}^{2}(6)\left(\bmod p^{2}\right) .
\end{aligned}
$$

From (2.12) and (2.13), the desired result is completed. The proof of others are obtained similar to the proof of (2.10).

Lemma 5. Let $p$ be an odd prime number. For $d, t \in \mathbb{Z}$,

$$
Q_{t(p-1)+d}=\left(\frac{p}{3}\right)^{t} Q_{\delta t+d}
$$

where (-) Jacobi symbol and $\delta=\frac{1-\left(\frac{p}{3}\right)}{2}$. 
Proof. Using $\alpha^{6 k}=\beta^{6 k}=1$ for $k \in \mathbb{Z}$, the proof is clearly obtained from the Binet formula of the sequence $\left\{Q_{n}\right\}$.

Theorem 1. Let $p>3$ be an odd prime and let $d$ and $t$ be any positive integers.

(1) For odd $t$ and $\left(\frac{t}{3}\right) \neq 0$,

$$
\begin{aligned}
6 \sum_{k=1}^{p-1} H_{k} Q_{t k+d} \equiv & \left(2\left(\frac{p}{3}\right) Q_{d-\delta t}+Q_{d+t}\right) \\
& \times\left(q_{p}(2)-\frac{1}{2} p q_{p}^{2}(2)+q_{p}(3)-\frac{1}{2} p q_{p}^{2}(3)-q_{p}(6)+\frac{1}{2} p q_{p}^{2}(6)\right) \\
& +\frac{p B_{p-2}\left(\frac{1}{3}\right)}{p-2}\left(\left(\frac{p}{3}\right) Q_{d+t}+Q_{d-\delta t}\right)\left(\bmod p^{2}\right)
\end{aligned}
$$

(2) For even $t$ and $\left(\frac{t}{3}\right) \neq 0$,

$-3 \sum_{k=1}^{p-1} H_{k} Q_{t k+d}$

$\equiv \frac{p B_{p-2}\left(\frac{1}{3}\right)}{3(p-2)} Q_{d+(2-2 \delta) t}+\frac{3}{2}\left(\frac{p}{3}\right) Q_{d+2 \delta t}\left(q_{p}(3)-\frac{1}{2} p q_{p}^{2}(3)\right)\left(\bmod p^{2}\right)$.

(3) For $\left(\frac{t}{3}\right)=0$,

$\frac{1}{Q_{d}} \sum_{k=1}^{p-1} H_{k} Q_{t k+d} \equiv 1-\Delta(t)\left(1+q_{p}(2)\right)$

$$
-p\left(1-\Delta(t)\left(1+\frac{q_{p}^{2}(2)}{2}\right)\right)\left(\bmod p^{2}\right)
$$

where $\Delta(t)=\frac{1-(-1)^{t}}{2}, \delta=\frac{1-\left(\frac{p}{3}\right)}{2}$, and Fermat quotient $q_{p}(a)=\frac{a^{p-1}-1}{p}$. 
Proof. By the Binet formula of the sequence $\left\{Q_{n}\right\}$, we observe that

$$
\begin{aligned}
\sum_{k=1}^{p-1} H_{k} Q_{t k+d}= & \sum_{k=1}^{p-1} Q_{t k+d} \sum_{j=1}^{k} \frac{1}{j}=\sum_{j=1}^{p-1} \frac{1}{j} \sum_{k=j}^{p-1} Q_{t k+d} \\
= & \sum_{j=1}^{p-1} \frac{1}{j}\left\{\sum_{k=0}^{p-1} Q_{t k+d}-\sum_{k=0}^{j-1} Q_{t k+d}\right\} \\
= & \sum_{j=1}^{p-1} \frac{1}{j}\left\{\alpha^{d} \frac{b-a \beta}{\alpha-\beta}\left(\sum_{k=0}^{p-1} \alpha^{t k}-\sum_{k=0}^{j-1} \alpha^{t k}\right)\right. \\
& \left.-\beta^{d} \frac{b-a \alpha}{\alpha-\beta}\left(\sum_{k=0}^{p-1} \beta^{t k}-\sum_{k=0}^{j-1} \beta^{t k}\right)\right\} \\
= & \left(\frac{b-a \beta}{\alpha-\beta} \frac{\alpha^{t p+d}}{\alpha^{t}-1}-\frac{b-a \alpha}{\alpha-\beta} \frac{\beta^{t p+d}}{\beta^{t}-1}\right) \sum_{j=1}^{p-1} \frac{1}{j} \\
& -\sum_{j=1}^{p-1} \frac{1}{j}\left(\alpha^{d} \frac{b-a \beta}{\alpha-\beta} \frac{\alpha^{t j}}{\alpha^{t}-1}-\beta^{d} \frac{b-a \alpha}{\alpha-\beta} \frac{\beta^{t j}}{\beta^{t}-1}\right) .
\end{aligned}
$$

(1) For $t=6 n+1$ and $t=6 n+5, n \in \mathbb{Z}$, we have $\alpha^{t}+\beta^{t}=1$. Thus,

$$
\begin{aligned}
\sum_{k=1}^{p-1} H_{k} Q_{t k+d} & \\
= & \left(\frac{b-a \beta}{\alpha-\beta}\left(\alpha^{t(p-1)+d}-\alpha^{t p+d}\right)-\frac{b-a \alpha}{\alpha-\beta}\left(\beta^{t(p-1)+d}-\beta^{t p+d}\right)\right) \sum_{j=1}^{p-1} \frac{1}{j} \\
& -\sum_{j=1}^{p-1} \frac{1}{j}\left(\alpha^{d} \frac{b-a \beta}{\alpha-\beta}\left(\alpha^{t(j-1)}-\alpha^{t j}\right)-\beta^{d} \frac{b-a \alpha}{\alpha-\beta}\left(\beta^{t(j-1)}-\beta^{t j}\right)\right) .
\end{aligned}
$$

By the Binet formula of the sequence $\left\{Q_{n}\right\}$, we get 


$$
\sum_{k=1}^{p-1} H_{k} Q_{t k+d}=\left(Q_{t(p-1)+d}-Q_{t p+d}\right) H_{p-1}-\sum_{j=1}^{p-1} \frac{1}{j}\left(Q_{t(j-1)+d}-Q_{t j+d}\right) .
$$

From the equality in (2.1) and the congruence in (1.1), we write

$$
\begin{aligned}
\sum_{k=1}^{p-1} H_{k} Q_{t k+d} & \\
= & Q_{t(p-2)+d} H_{p-1}-\sum_{j=1}^{p-1} \frac{Q_{t(j-2)+d}}{j} \equiv-\sum_{j=1}^{p-1} \frac{Q_{t(j-2)+d}}{j} \\
= & Q_{d-t} \sum_{\substack{k=1 \\
k \equiv 1(\bmod 3)}}^{p-1} \frac{(-1)^{k}}{k}+Q_{d} \sum_{k=1}^{p-1} \frac{(-1)^{k+1}}{k} \\
& +Q_{d+t} \sum_{\substack{k=1 \\
k \equiv 0(\bmod 3)}}^{p-1} \frac{(-1)^{k}}{k}\left(\bmod p^{2}\right) .
\end{aligned}
$$

For $\left(\frac{p}{3}\right)=1$, with the help of the congruences in (2.9), (2.10), and (2.11), we have

$$
\begin{aligned}
\sum_{k=1}^{p-1} H_{k} Q_{t k+d} \equiv & Q_{d-t} \sum_{k=1}^{\left\lfloor\frac{p}{3}\right\rfloor} \frac{(-1)^{k+1}}{p-3 k}-Q_{d} \sum_{k=1}^{\left\lfloor\frac{p}{3}\right\rfloor} \frac{(-1)^{k}}{p-3 k+1}-Q_{d+t} \sum_{k=1}^{\left\lfloor\frac{p}{3}\right\rfloor} \frac{(-1)^{k}}{p-3 k+2} \\
\equiv & \left(q_{p}(2)-\frac{1}{2} p q_{p}^{2}(2)\right)\left(Q_{d}-\frac{2}{3} Q_{d-t}-\frac{1}{2} Q_{d+t}\right) \\
& +\frac{1}{3}\left(q_{p}(3)-\frac{1}{2} p q_{p}^{2}(3)-q_{p}(6)+\frac{1}{2} p q_{p}^{2}(6)\right)\left(Q_{d}+\frac{1}{2} Q_{d+t}\right) \\
& +\frac{p B_{p-2}\left(\frac{1}{3}\right)}{6(p-2)}\left(Q_{d+t}+Q_{d}\right)\left(\bmod p^{2}\right) .
\end{aligned}
$$

Applying Lemma 1 with $n=0$, we can rewrite 


$$
\begin{aligned}
& \sum_{k=1}^{p-1} H_{k} Q_{t k+d} \\
& \equiv \frac{1}{6}\left(q_{p}(2)-\frac{1}{2} p q_{p}^{2}(2)+q_{p}(3)-\frac{1}{2} p q_{p}^{2}(3)-q_{p}(6)+\frac{1}{2} p q_{p}^{2}(6)\right)\left(2 Q_{d}+Q_{d+t}\right) \\
& \quad+\frac{p B_{p-2}\left(\frac{1}{3}\right)}{6(p-2)}\left(Q_{d+t}+Q_{d}\right)\left(\bmod p^{2}\right)
\end{aligned}
$$

Similarly, for $\left(\frac{p}{3}\right)=-1$, it is seen that

$$
\begin{aligned}
& \sum_{k=1}^{p-1} H_{k} Q_{t k+d} \\
& \equiv-\frac{1}{6}\left(q_{p}(2)-\frac{1}{2} p q_{p}^{2}(2)+q_{p}(3)-\frac{1}{2} p q_{p}^{2}(3)-q_{p}(6)+\frac{1}{2} p q_{p}^{2}(6)\right)\left(2 Q_{d-t}-Q_{d+t}\right) \\
& \quad-\frac{p B_{p-2}\left(\frac{1}{3}\right)}{6(p-2)}\left(Q_{d+t}+Q_{d-t}\right)\left(\bmod p^{2}\right) .
\end{aligned}
$$

Thus, by (2.15) and (2.16), we have completed the proof of Theorem 1 (1).

(2) For $t=6 n+2$ and $t=6 n+4, n \in \mathbb{Z}$, we have $\alpha^{t}+\beta^{t}=-1$. By the Binet formula of the sequence $\left\{Q_{n}\right\}$, we write

$$
\begin{aligned}
3 \sum_{k=1}^{p-1} H_{k} Q_{t k+d} & \\
= & \sum_{j=1}^{p-1} \frac{1}{j}\left(\alpha^{d} \frac{b-\alpha \beta}{\alpha-\beta}\left(\alpha^{t(p-1)}-\alpha^{t p}\right)-\beta^{d} \frac{b-a \alpha}{\alpha-\beta}\left(\beta^{t(p-1)}-\beta^{t p}\right)\right) \\
& -\sum_{j=1}^{p-1} \frac{1}{j}\left(\alpha^{d} \frac{b-a \beta}{\alpha-\beta}\left(\alpha^{t(j-1)}-\alpha^{t j}\right)-\beta^{d} \frac{b-a \alpha}{\alpha-\beta}\left(\beta^{t(j-1)}-\beta^{t j}\right)\right) \\
= & \left(Q_{t(p-1)+d}-Q_{t p+d}\right) H_{p-1}-\sum_{j=1}^{p-1} \frac{1}{j}\left(Q_{t(j-1)+d}-Q_{t j+d}\right) .
\end{aligned}
$$


From the congruence in (1.1), we have

$$
\begin{aligned}
-3 \sum_{k=1}^{p-1} H_{k} Q_{t k+d} \equiv & \sum_{j=1}^{p-1} \frac{1}{j}\left(Q_{t(j-1)+d}-Q_{t j+d}\right) \\
= & \sum_{\substack{k=1 \\
k \equiv 1(\bmod 3)}}^{p-1} \frac{1}{k}\left(Q_{d}-Q_{d+t}\right)+\sum_{\substack{k=1 \\
k \equiv 2(\bmod 3)}}^{p-1} \frac{1}{k}\left(Q_{d+t}-Q_{d+2 t}\right) \\
& +\sum_{\substack{k=1 \\
k \equiv 0(\bmod 3)}}^{p-1} \frac{1}{k}\left(Q_{d+2 t}-Q_{d}\right)\left(\bmod p^{2}\right) .
\end{aligned}
$$

For $\left(\frac{p}{3}\right)=1$,

$$
\begin{aligned}
-3 \sum_{k=1}^{p-1} H_{k} Q_{t k+d} \equiv & \sum_{k=1}^{\left\lfloor\frac{p}{3}\right\rfloor} \frac{1}{p-3 k}\left(Q_{d}-Q_{d+t}\right) \\
& +\sum_{k=1}^{\left\lfloor\frac{p}{3}\right\rfloor} \frac{1}{p-3 k+1}\left(Q_{d+t}-Q_{d+2 t}\right) \\
& +\sum_{k=1}^{\left\lfloor\frac{p}{3}\right\rfloor} \frac{1}{p-3 k+2}\left(Q_{d+2 t}-Q_{d}\right)\left(\bmod p^{2}\right)
\end{aligned}
$$

By the congruences in (1.6), (2.3), and (2.4), we get

$$
\begin{aligned}
-3 \sum_{k=1}^{p-1} H_{k} Q_{t k+d} \equiv & \left(q_{p}(3)-\frac{1}{2} p q_{p}^{2}(3)\right)\left(Q_{d}-\frac{1}{2}\left(Q_{d+t}+Q_{d+2 t}\right)\right) \\
& +\frac{p B_{p-2}\left(\frac{1}{3}\right)}{9(p-2)}\left(2 Q_{d+2 t}-Q_{d+t}-Q_{d}\right)\left(\bmod p^{2}\right)
\end{aligned}
$$

Using $Q_{t+d}+Q_{d}=-Q_{2 t+d}$ in (2.1), it is clearly seen that 


$$
\begin{aligned}
-3 \sum_{k=1}^{p-1} H_{k} Q_{t k+d} \equiv & \frac{3}{2}\left(q_{p}(3)-\frac{1}{2} p q_{p}^{2}(3)\right) Q_{d} \\
& +\frac{p B_{p-2}\left(\frac{1}{3}\right)}{3(p-2)} Q_{d+2 t}\left(\bmod p^{2}\right)
\end{aligned}
$$

Similarly, for $\left(\frac{p}{3}\right)=-1$,

$$
\begin{aligned}
-3 \sum_{k=1}^{p-1} H_{k} Q_{t k+d} \equiv & -\frac{3}{2}\left(q_{p}(3)-\frac{1}{2} p q_{p}^{2}(3)\right) Q_{d+2 t} \\
& +\frac{p B_{p-2}\left(\frac{1}{3}\right)}{3(p-2)} Q_{d}\left(\bmod p^{2}\right)
\end{aligned}
$$

By (2.17) and (2.18), the proof is completed.

(3) For $t=6 n$ and $t=6 n+3, n \in \mathbb{Z}$, it is known that $Q_{t n+d}=$ $(-1)^{t n} Q_{d}$ in (2.1). Using $\sum_{k=1}^{n} H_{k}=(n+1) H_{n}-n$ and $\sum_{k=1}^{n}(-1)^{k} H_{k}$ $=\left(\frac{(-1)^{n}-1}{2}\right) H_{n}+\frac{1}{2} H_{\lfloor n / 2\rfloor}$, we write

$$
\begin{aligned}
\sum_{k=1}^{p-1} H_{k} Q_{t k+d} & =Q_{d} \sum_{k=1}^{p-1}(-1)^{t k} H_{k} \\
& =Q_{d}\left\{\begin{array}{cc}
p H_{p-1}-p+1, & \text { if } t \text { is even } \\
\frac{1}{2} H_{\frac{p-1}{2}}, & \text { if } t \text { is odd }
\end{array} .\right.
\end{aligned}
$$

From the congruences $\sum_{j=1}^{(p-1) / 2} \frac{1}{j} \equiv-2\left(q_{p}(2)-\frac{1}{2} p q_{p}^{2}(2)\right)\left(\bmod p^{2}\right)$ in [1] and $H_{p-1} \equiv 0\left(\bmod p^{2}\right)$, we obtain 


$$
\sum_{k=1}^{p-1} H_{k} Q_{t k+d} \equiv Q_{d}\left\{\begin{array}{cc}
1-p, & \text { if } t \text { is even } \\
-q_{p}(2)+\frac{1}{2} p q_{p}^{2}(2), & \text { if } t \text { is odd }
\end{array}\left(\bmod p^{2}\right) .\right.
$$

So the proof is completed.

Theorem 2. Let $p$ be an odd prime and $d$ and $t$ be any positive integers.

(1) For odd $t$ and $\left(\frac{t}{3}\right) \neq 0$,

$-6 \sum_{k=1}^{p-1} k H_{k} Q_{t k+d}$

$\equiv\left(q_{p}(2)-\frac{1}{2} p q_{p}^{2}(2)+q_{p}(3)-\frac{1}{2} p q_{p}^{2}(3)-q_{p}(6)+\frac{1}{2} p q_{p}^{2}(6)\right)\left(Q_{d}-2\left(\frac{p}{3}\right) Q_{d+(2-\delta) t}\right)$

$+\left(\frac{p B_{p-2}\left(\frac{1}{3}\right)}{p-2}+6 \delta\right)\left(Q_{d}-\left(\frac{p}{3}\right) Q_{d+(2-\delta) t}\right)\left(\bmod p^{2}\right)$.

(2) For even $t$ and $\left(\frac{t}{3}\right) \neq 0$,

$-3 \sum_{k=1}^{p-1} k H_{k} Q_{t k+d} \equiv \frac{1}{2}\left(q_{p}(3)-\frac{1}{2} p q_{p}^{2}(3)\right)\left(Q_{d+(\delta+1) t}-Q_{d}\right)$

$-\left(\frac{p}{3}\right)\left(\frac{p B_{p-2}\left(\frac{1}{3}\right)}{9(p-2)}-\delta\right)\left(Q_{d+(2-\delta) t}-Q_{d}\right)\left(\bmod p^{2}\right)$.

(3) For $\left(\frac{t}{3}\right)=0$,

$$
\begin{aligned}
\frac{1}{Q_{d}} \sum_{k=1}^{p-1} k H_{k} Q_{t k+d} \equiv & \frac{p}{4}\left(3-\Delta(t)\left(3+q_{p}^{2}(2)\right)\right) \\
& -\frac{1}{2}\left(1-\Delta(t)\left(1+q_{p}(2)\right)\right)\left(\bmod p^{2}\right),
\end{aligned}
$$

where $\delta, \Delta(t), q_{p}(a)$ can be seen as in Theorem 1 . 
Proof. By the Binet formula of the sequence $\left\{Q_{n}\right\}$, we have

$$
\begin{aligned}
\sum_{k=1}^{p-1} k H_{k} Q_{t k+d} & \\
= & \sum_{k=1}^{p-1} k Q_{t k+d} \sum_{j=1}^{k} \frac{1}{j}=\sum_{j=1}^{p-1} \frac{1}{j} \sum_{k=j}^{p-1} k Q_{t k+d} \\
= & \sum_{j=1}^{p-1} \frac{1}{j}\left\{\sum_{k=0}^{p-1} k Q_{t k+d}-\sum_{k=0}^{j-1} k Q_{t k+d}\right\} \\
= & \sum_{j=1}^{p-1} \frac{1}{j}\left\{\alpha^{d} \frac{b-a \beta}{\alpha-\beta}\left(\sum_{k=0}^{p-1} k \alpha^{t k}-\sum_{k=0}^{j-1} k \alpha^{t k}\right)\right. \\
& \left.-\beta^{d} \frac{b-a \alpha}{\alpha-\beta}\left(\sum_{k=0}^{p-1} k \beta^{t k}-\sum_{k=0}^{j-1} k \beta^{t k}\right)\right\} .
\end{aligned}
$$

Using the sum $\sum_{k=0}^{n} k x^{k} y^{n-k}=\frac{(n+1) x^{n+1}(x-y)-x\left(x^{n+1}-y^{n+1}\right)}{(x-y)^{2}}$, we write

$$
\begin{aligned}
\sum_{k=1}^{p-1} k H_{k} Q_{t k+d} & \\
= & \sum_{j=1}^{p-1} \frac{1}{j}\left(\alpha^{d} \frac{b-a \beta}{\alpha-\beta} \frac{\left((p-1) \alpha^{t(p+1)}-p \alpha^{t p}\right)}{\left(\alpha^{t}-1\right)^{2}}\right. \\
& \left.-\beta^{d} \frac{b-a \alpha}{\alpha-\beta} \frac{\left((p-1) \beta^{t(p+1)}-p \beta^{t p}\right)}{\left(\beta^{t}-1\right)^{2}}\right) \\
& -\sum_{j=1}^{p-1} \frac{1}{j}\left(\alpha^{d} \frac{b-a \beta}{\alpha-\beta} \frac{\left((j-1) \alpha^{t(j+1)}-j \alpha^{t j}\right)}{\left(\alpha^{t}-1\right)^{2}}\right. \\
& \left.-\beta^{d} \frac{b-a \alpha}{\alpha-\beta} \frac{\left((j-1) \beta^{t(j+1)}-j \beta^{t j}\right)}{\left(\beta^{t}-1\right)^{2}}\right) .
\end{aligned}
$$


(1) For $t=6 n+1$ and $t=6 n+5, n \in \mathbb{Z}$, note that $\alpha^{t}+\beta^{t}=1$.

Thus,

$$
\begin{aligned}
\sum_{k=1}^{p-1} k H_{k} Q_{t k+d} & \\
= & \sum_{j=1}^{p-1} \frac{1}{j}\left(\alpha^{d} \frac{b-a \beta}{\alpha-\beta}\left(p \alpha^{t(p-1)}-(p-1) \alpha^{t p}\right)\right. \\
& \left.-\beta^{d} \frac{b-a \alpha}{\alpha-\beta}\left(p \beta^{t(p-1)}-(p-1) \beta^{t p}\right)\right) \\
& -\sum_{j=1}^{p-1} \frac{1}{j}\left(\alpha^{d} \frac{b-a \beta}{\alpha-\beta}\left(j \alpha^{t(j-1)}-(j-1) \alpha^{t j}\right)\right. \\
& \left.-\beta^{d} \frac{b-a \alpha}{\alpha-\beta}\left(j \beta^{t(j-1)}-(j-1) \beta^{t j}\right)\right),
\end{aligned}
$$

and by the Binet formula of the sequence $\left\{Q_{n}\right\}$, we get

$$
\begin{aligned}
\sum_{k=1}^{p-1} k H_{k} Q_{t k+d}= & \left(p Q_{t(p-1)+d}-(p-1) Q_{t p+d}\right) H_{p-1} \\
& -\sum_{j=1}^{p-1} \frac{1}{j}\left(j Q_{t(j-1)+d}-(j-1) Q_{t j+d}\right) .
\end{aligned}
$$

Using (2.14) for odd $t$ and the congruence $H_{p-1} \equiv 0\left(\bmod p^{2}\right)$, we write

$$
\begin{aligned}
\sum_{k=1}^{p-1} k H_{k} Q_{t k+d} & \equiv-\sum_{j=1}^{p-1} \frac{1}{j}\left(j Q_{t(j-1)+d}-(j-1) Q_{t j+d}\right) \\
& =-\sum_{j=1}^{p-1}\left(Q_{t(j-1)+d}-Q_{t j+d}\right)-\sum_{j=1}^{p-1} \frac{Q_{t j+d}}{j} \\
& =-Q_{d}+Q_{t(p-1)+d}-\sum_{j=1}^{p-1} \frac{Q_{t j+d}}{j}
\end{aligned}
$$




$$
\begin{aligned}
=-Q_{d}+\left(\frac{p}{3}\right) Q_{\delta t+d}+Q_{d+t} \sum_{\substack{k=1 \\
k \equiv 1(\bmod 3)}}^{p-1} \frac{(-1)^{k}}{k} \\
+Q_{d+2 t} \sum_{\substack{k=1 \\
k \equiv 2(\bmod 3)}}^{p-1} \frac{(-1)^{k+1}}{k}+Q_{d} \sum_{\substack{k=1 \\
k \equiv 0(\bmod 3)}}^{p-1} \frac{(-1)^{k+1}}{k}\left(\bmod p^{2}\right) .
\end{aligned}
$$

For $\left(\frac{p}{3}\right)=1$, with the help of the congruences in (2.9), (2.10), and (2.11), we give

$$
\begin{aligned}
\sum_{k=1}^{p-1} k H_{k} Q_{t k+d} \equiv & -Q_{d}+Q_{d}+Q_{d+t} \sum_{k=1}^{\left\lfloor\frac{p}{3}\right\rfloor} \frac{(-1)^{k+1}}{p-3 k} \\
& -Q_{d+2 t} \sum_{k=1}^{\left\lfloor\frac{p}{3}\right\rfloor} \frac{(-1)^{k}}{p-3 k+1}-Q_{d} \sum_{k=1}^{\left\lfloor\frac{p}{3}\right\rfloor} \frac{(-1)^{k+1}}{p-3 k+2} \\
\equiv & -\left(q_{p}(2)-\frac{1}{2} p q_{p}^{2}(2)\right)\left(\frac{2}{3} Q_{d+t}-\frac{1}{2} Q_{d}-Q_{d+2 t}\right) \\
& -\frac{1}{6}\left(q_{p}(3)-\frac{1}{2} p q_{p}^{2}(3)-q_{p}(6)+\frac{1}{2} p q_{p}^{2}(6)\right)\left(Q_{d}-2 Q_{d+2 t}\right) \\
& -\frac{p B_{p-2}\left(\frac{1}{3}\right)}{6(p-2)}\left(Q_{d}-Q_{d+2 t}\right)\left(\bmod p^{2}\right) .
\end{aligned}
$$

Using Lemma 1 with $n=0$, the desired result is obtained.

Similarly, for $\left(\frac{p}{3}\right)=-1$, using the congruences in (2.9), (2.10), and (2.11), we give 


$$
\begin{aligned}
& \sum_{k=1}^{p-1} k H_{k} Q_{t k+d} \\
\equiv & \frac{1}{6}\left(q_{p}(2)-\frac{1}{2} p q_{p}^{2}(2)+q_{p}(3)-\frac{1}{2} p q_{p}^{2}(3)-q_{p}(6)+\frac{1}{2} p q_{p}^{2}(6)\right)\left(-Q_{d}-2 Q_{d+t}\right) \\
+ & \left(\frac{p B_{p-2}\left(\frac{1}{3}\right)}{6(p-2)}-1\right)\left(Q_{d}+Q_{d+t}\right)\left(\bmod p^{2}\right) .
\end{aligned}
$$

Thus, this completes the proof of Theorem 2 (1).

(2) For $t=6 n+2$ and $t=6 n+4, n \in \mathbb{Z}$, since $\alpha^{t}+\beta^{t}=-1$, we write

$$
\begin{aligned}
3 \sum_{k=1}^{p-1} k H_{k} & Q_{t k+d} \\
= & \sum_{j=1}^{p-1} \frac{1}{j}\left(\alpha^{d} \frac{b-a \beta}{\alpha-\beta}\left(p \alpha^{t(p-1)}-(p-1) \alpha^{t p}\right)\right. \\
& \left.-\beta^{d} \frac{b-a \alpha}{\alpha-\beta}\left(p \beta^{t(p-1)}-(p-1) \beta^{t p}\right)\right) \\
& -\sum_{j=1}^{p-1} \frac{1}{j}\left(\alpha^{d} \frac{b-a \beta}{\alpha-\beta}\left(j \alpha^{t(j-1)}-(j-1) \alpha^{t j}\right)\right. \\
& \left.-\beta^{d} \frac{b-a \alpha}{\alpha-\beta}\left(j \beta^{t(j-1)}-(j-1) \beta^{t j}\right)\right) .
\end{aligned}
$$

By the Binet formula of the sequence $\left\{Q_{n}\right\}$, we get

$$
\begin{aligned}
3 \sum_{k=1}^{p-1} k H_{k} Q_{t k+d}= & \left(p Q_{t(p-1)+d}-(p-1) Q_{t p+d}\right) H_{p-1} \\
& -\sum_{j=1}^{p-1} \frac{1}{j}\left(j Q_{t(j-1)+d}-(j-1) Q_{t j+d}\right) .
\end{aligned}
$$

From the congruence in (1.1), using (2.14) for even $t$, we write 


$$
\begin{aligned}
3 \sum_{k=1}^{p-1} k H_{k} Q_{t k+d} & \\
\equiv & -\sum_{j=1}^{p-1} \frac{1}{j}\left(j Q_{t(j-1)+d}-(j-1) Q_{t j+d}\right) \\
= & -\sum_{j=1}^{p-1}\left(Q_{t(j-1)+d}-Q_{t j+d}\right)-\sum_{j=1}^{p-1} \frac{Q_{t j+d}}{j} \\
= & -Q_{d}+Q_{t(p-1)+d}-\sum_{j=1}^{p-1} \frac{Q_{t j+d}}{j} \\
= & -Q_{d}+\left(\frac{p}{3}\right) Q_{\delta t+d}-Q_{d+t} \sum_{\substack{k=1 \\
k=1(\bmod 3)}}^{p-1} \frac{1}{k} \\
& -Q_{d+2 t} \sum_{\substack{k=1 \\
k \equiv 2(\bmod 3)}}^{p-1} \sum_{k=0(\bmod 3)}^{p-1} \frac{1}{k}\left(\bmod p^{2}\right) .
\end{aligned}
$$

For $\left(\frac{p}{3}\right)=1$, then

$$
\begin{aligned}
3 \sum_{k=1}^{p-1} k H_{k} Q_{t k+d} \equiv & -Q_{d+t} \sum_{k=1}^{\left\lfloor\frac{p}{3}\right\rfloor} \frac{1}{p-3 k}-Q_{d+2 t} \sum_{k=1}^{\left\lfloor\frac{p}{3}\right\rfloor} \frac{1}{p-3 k+1} \\
& -Q_{d} \sum_{k=1}^{\left\lfloor\frac{p}{3}\right\rfloor} \frac{1}{p-3 k+2}\left(\bmod p^{2}\right) .
\end{aligned}
$$

By the congruences in (1.6), (2.3), and (2.4), it is clearly seen that

$$
\begin{aligned}
3 \sum_{k=1}^{p-1} k H_{k} Q_{t k+d} \equiv & -\frac{1}{2}\left(q_{p}(3)-\frac{1}{2} p q_{p}^{2}(3)\right)\left(Q_{d+t}-Q_{d}\right) \\
& +\frac{p B_{p-2}\left(\frac{1}{3}\right)}{9(p-2)}\left(Q_{d+2 t}-Q_{d}\right)\left(\bmod p^{2}\right) .
\end{aligned}
$$


Similarly, for $\left(\frac{p}{3}\right)=-1$,

$$
\begin{aligned}
3 \sum_{k=1}^{p-1} k H_{k} Q_{t k+d} \equiv & -\frac{1}{2}\left(q_{p}(3)-\frac{1}{2} p q_{p}^{2}(3)\right)\left(Q_{d+2 t}-Q_{d}\right) \\
& -\left(\frac{p B_{p-2}\left(\frac{1}{3}\right)}{9(p-2)}-1\right)\left(Q_{d+t}-Q_{d}\right)\left(\bmod p^{2}\right) .
\end{aligned}
$$

By (2.19) and (2.20), the proof is completed.

(3) For $t=6 n$ and $t=6 n+3, n \in \mathbb{Z}$, it is known that $Q_{t n+d}=$ $(-1)^{t n} Q_{d}$ in (2.1). Using the sums $\sum_{k=1}^{n} k H_{k}=\frac{n(n+1)}{2} H_{n}-\frac{n(n-1)}{4}$ and $\sum_{k=1}^{n}(-1)^{k} k H_{k}=(-1)^{n}\left(\frac{n}{2}+\frac{1}{4}\right) H_{n}-\frac{1}{4}\left(H_{\lfloor n / 2\rfloor}-H_{n}\right)+\left(\frac{(-1)^{n}-1}{4}\right)$, we write

$$
\begin{aligned}
\sum_{k=1}^{p-1} k H_{k} Q_{t k+d} & =Q_{d} \sum_{k=1}^{p-1}(-1)^{t k} k H_{k} \\
& \equiv Q_{d} \begin{cases}-\frac{p}{2} H_{p-1}+\frac{3 p-2}{4}, & \text { if } t \text { is even }\left(\bmod p^{2}\right) \\
\frac{p}{2} H_{p-1}-\frac{1}{4} H_{\frac{p-1}{2}}, & \text { if } t \text { is odd }\end{cases}
\end{aligned}
$$

Since the congruences, $\sum_{j=1}^{(p-1) / 2} \frac{1}{j} \equiv-2\left(q_{p}(2)-\frac{1}{2} p q_{p}^{2}(2)\right)\left(\bmod p^{2}\right)$ in [1] and $H_{p-1} \equiv 0\left(\bmod p^{2}\right)$, we have

$$
\sum_{k=1}^{p-1} k H_{k} Q_{t k+d} \equiv Q_{d}\left\{\begin{array}{cl}
\frac{3 p-2}{4}, & \text { if } t \text { is even }\left(\bmod p^{2}\right) . \\
\frac{1}{2}\left(q_{p}(2)-\frac{1}{2} p q_{p}^{2}(2)\right), & \text { if } t \text { is odd }
\end{array}\right.
$$

So the proof is completed. 


\section{References}

[1] E. Lehmer, On congruences involving Bernoulli numbers and the quotients of Fermat and Wilson, Ann. of Math. 39 (1938), 350-360.

[2] J. Wolstenholme, On certain properties of prime numbers, Quart. J. Math. 5 (1862), 35-39.

[3] W. Magnus, F. Oberhettinger and R. P. Soni, Formulas and Theorems for the Special Functions of Mathematical Physics (3rd Edition), Springer-Verlag, New York, (1966), 25-32.

[4] Z. H. Sun, Congruences for Bernoulli numbers and Bernoulli polynomials, Discrete Mathematics 163 (1997), 153-163.

[5] Z. H. Sun, Congruences concerning Bernoulli numbers and Bernoulli polynomials, Discrete Applied Mathematics 105 (2000), 193-223.

[6] Z. H. Sun, Congruences involving Bernoulli and Euler numbers, Journal of Number Theory 128(2) (2008), 280-312.

[7] Z. W. Sun and R. Tauraso, On some new congruences for Binomial coefficients, Int. Journal of Number Theory 7(3) (2011), 645-662.

[8] Z. W. Sun, On harmonic numbers and Lucas sequences, Publ. Math. Debrecen 8081-2 (2012), 25-41.

[9] Z. W. Sun, Arithmetic theory of harmonic numbers, Proc. Amer. Math. Soc. 140 (2012), 415-428. 